\title{
POET: A Model for Planetary Orbital Evolution due to Tides on Evolving Stars
}

\author{
Kaloyan Penev \\ Department of Astrophysical Sciences, 4 Ivy Lane, Peyton Hall, Princeton University, Princeton, NJ \\ $08544, U S A$ \\ Michael Zhang \\ Department of Astrophysical Sciences, 4 Ivy Lane, Peyton Hall, Princeton University, Princeton, NJ \\ 08544, USA \\ Brian Jackson \\ Carnegie DTM, 5241 Broad Branch Road, NW, Washington, DC 20015-1305, USA
}

\begin{abstract}
We make publicly available an efficient, versatile, easy to use and extend tool for calculating the evolution of circular aligned planetary orbits due to the tidal dissipation in the host star. This is the first model to fully account for the evolution of the angular momentum of the stellar convective envelope by the tidal coupling, the transfer of angular momentum between the stellar convective and radiative zones, the effects of the stellar evolution on the tidal dissipation efficiency and stellar core and envelope spins, the loss of stellar convective zone angular momentum to a magnetically launched wind and frequency dependent tidal dissipation. This is only a first release and further development is under way to allow calculating the evolution of inclined and eccentric orbits, with the latter including the tidal dissipation in the planet and its feedback on planetary structure. Considerable effort has been devoted to providing extensive documentation detailing both the usage and the complete implementation details, in order to make it as easy as possible for independent groups to use and/or extend the code for their purposes. POET represents a significant improvement over some previous models for planetary tidal evolution and so has many astrophysical applications. In this article, we describe and illustrate several key examples.
\end{abstract}

Subject headings: convection — planet-star interactions — stars: interiors — stars: rotation — stars: winds, outflows - turbulence

\section{Introduction}

The very first extrasolar (or exo-) planets discovered were planets with orbital periods less than a few days, and the increasing number of such planets over the last few decades shows they are not flukes - close-in exoplanets represent a robust outcome of planet formation and evolution. Moreover, owing to observational biases, close-in exoplanets dominate observational constraints on planetary composition, internal structure, meteo- rology, etc., and so resolving the severe challenges they pose to theories of formation is critical for extrapolating these constraints to all planets.

Among other influences, tidal interactions between the planets and their host stars shape the planetary population, and a long list of studies have investigated the effects of tides in extrasolar systems, including Sandquist et al. (2002); Sasselov (2003); Levrard et al. (2009); Jackson et al. (2009); Debes \& Jackson (2010); 
Jackson et al. (2010); Penev et al. (2012); Pepper et al. (2013); Collins et al. (2013); Zhang \& Penev (2013). Close-in exoplanets likely formed in more distant ( $\gtrsim 1 \mathrm{AU})$, nearly circular orbits, embedded in a protoplanetary disk well-aligned with the stellar equator (Lin et al. 1996, although see Batygin \& Adams 2013), and there are two standard scenarios for bringing them into close-in orbits: (1) dynamical excitation of orbital eccentricity, followed by tidal evolution and (2) gas disk migration. Under scenario (1), gravitational excitations scattered the planets into highly eccentric orbits, with pericenter distances small enough for tidal interaction with the host star to bring the planets in (Rasio \& Ford 1996; Weidenschilling \& Marzari 1996; Fabrycky \& Tremaine 2007; Nagasawa et al. 2008; Lithwick \& Wu 2013; Valsecchi \& Rasio 2014). Under scenario (2), transfer of angular momentum from the planet's orbit to the protoplanetary disk caused rapid inward migration (e.g. Chambers 2009). Lin et al. (1996) suggested the migration would cease as the planet entered a clearing in the gas disk near the host star, parking the planet in a close-in orbit (at least temporarily). Orbital decay from tides may then continue, as long as the rotation of the host star lags behind the planet's orbital motion. Levrard et al. (2009) showed that most close-in gas giants are unstable against tidal decay, and Jackson et al. (2009) showed their orbital distribution is consistent with complete orbital decay and planetary disruption. More recently, Teitler \& Königl (2014) showed that the distribution of the orbital and stellar rotation periods for short period planets discoverd by the Kepler space telescope is consistent with tidal ingestion of planets, which contributes to spinning up the host stars. A related topic, Carlberg et al. (2009) showed that tidal decay during the post-main sequence can even remove gas giants in more distant orbits resembling Jupiter's.

Thus, tidal evolution spans from the pre- to post-main sequence, during which time the host stars undergo significant evolution as well, and, as we show, this stellar evolution can have dramatic consequences for the orbital evolution of close-in exoplanets. However, all previous tidal studies incorporated simplifying assumptions that exclude important effects. To address this short-coming, we have developed and made publicly available the Planetary Orbital Evolution due to Tides (P.O.E.T.) model. Using grids of stellar evolution tracks from the Yale Rotating Stellar Evolution Code - YREC - (Demarque et al. 2008), P.O.E.T. is the only presently available model that includes the coupling between stellar and tidal evolution.

Consequently, P.O.E.T. has a wide range of capabilities. For example, P.O.E.T. tracks the stellar spin evolution, including angular momentum loss from the stellar wind in a self-consistent way. As we discuss in Section 5 (see Fig. 2), this can have profound consequences for the computed evolution. Unlike simplified models which ignore stellar evolution P.O.E.T. is capable of computing the evolution of orbits around pre- and post-main sequence stars. An example of this is again given in Section 5] (see Fig. [3).

Since comparison of model predictions of tidal evolution to observations is usually statistical in nature, we have made the code efficient, enabling millions of model calculations with modest computational resources in a short time. Finally, ease of use has been a primary consideration. To that end, we provide two interfaces to the tidal evolution calculator: a command line tool and a python module, and both interfaces provide access to all model parameters. We have also written extensive documentation 1 .

Of course, like any numerical model, P.O.E.T. has limitations. The most obvious in the present version is the assumption that the planetary orbit is circular and aligned with the stellar equator. Development is underway to relax these assumptions for future versions of P.O.E.T.. Of course we can never hope to develop a completely universal tool, so we have devoted considerable effort to document the code and make it as easy as possible for users to adapt it to their applications.

In Sections 2 and 3, we briefly detail the physical and numerical schemes employed by P.O.E.T., respectively. In Section 4, we discuss the grid of $Y R E C$ stellar evolution calculations. In Section 5. we present example calculations, showing the importance of including the stellar rotation and internal evolution. Finally, in Section 6, we discuss limitations and planned improvements to the code. Appendix A explains how to install P.O.E.T. Ap-

1 http://www.astro.princeton.edu/ kpenev/tidal_orbital_evolution/ 
pendices $\mathrm{B}$ and $\mathrm{C}$ provide a more detailed description of the numerical scheme.

\section{Physical Model}

We define our notation up front (see text for details):

$M_{*}$ mass of the star

$R_{*}$ radius of the star

$m_{p}$ mass of the planet

$r_{p}$ radius of the planet

$Q_{*}$ modified tidal quality factor of the star (cf. Ogilvie \& Lin 2007)

$a$ semimajor axis of the orbit

$\omega_{\text {surf }}$ angular velocity of the stellar surface $\left(\omega_{\text {conv }}\right.$ for low mass stars and the solid body rotation for high mass stars)

$\omega_{\text {conv/rad }}$ angular velocity of the stellar convective/radiative zone

$\omega_{\text {orb }}$ orbital angular velocity

$I_{\text {conv/rad/* moment of inertia of the stellar convec- }}$ tive/radiative zone or the entire star.

$L_{\text {conv/rad }}$ angular momentum of the stellar convective/radiative zone

$L_{*}$ angular momentum of an entire high mass star

$K$ parameter giving the strength of the magnetic wind of the star

$\omega_{\text {sat }}$ stellar surface angular velocity at which the magnetic wind saturates

$\tau_{c}$ stellar core-envelope coupling timescale

$M_{\mathrm{rad}}$ mass of the radiative core for low mass stars

$R_{\text {rad }}$ radius of the radiative-convective boundary in low mass stars

$M_{*}^{\text {crit }}$ stellar mass below which stars are split into a radiative core and a convective envelope and above which they are treated as solid bodies, ignoring the tiny surface convective zone.
Our model computes the secular evolution of a planet-star system as its semi-major axis evolves due to the dissipation of the tides raised by the planet on the star. In fact the dissipation of the tides raised on the planet by the star may also be important. However, since the angular momentum of the planetary spin is very small compared to the angular momenta of the orbit or the star, the planet's rotation is synchronized quickly with the orbit. For an eccentric orbit, this would still lead to time dependent tides on the planet, however, our model currently assumes circular orbits. As a result, once the planet's rotation is synchronized to the orbit the planetary tides are stationary and not subject to dissipation.

Under these assumptions, the evolution of the semi-major axis is given by (Goldreich 1963; Kaula 1968; Jackson et al. 2008):

$$
\dot{a}=\operatorname{sign}\left(\omega_{\text {surf }}-\omega_{\text {orb }}\right) \frac{9}{2} \sqrt{\frac{G}{a M_{*}}}\left(\frac{R_{*}}{a}\right)^{5} \frac{m_{p}}{Q_{*}}
$$

Where $\operatorname{sign}\left(\omega_{\text {surf }}-\omega_{\text {orb }}\right)$ takes the value 1 when the stellar surface is spinning faster than the planet and -1 when it is spinning slower.

This expression neglects the planet's mass relative to the star's mass, a perfectly reasonable assumption, given the uncertainties, and indeed the lack of a good physical model for $Q_{*}$.

Angular momentum conservation requires that any angular momentum gained or lost by the orbit is taken from or added to the star, changing its spin. Simple arithmetic shows that the rate at which angular momentum is deposited into the star due to the orbit evolving is given by:

$$
\dot{L}_{\text {tide }} \equiv-\frac{1}{2} m_{p} M_{*} \sqrt{\frac{G}{a\left(M_{*}+m_{p}\right)}} \dot{a}
$$

Here we do not neglect the mass of the planet relative to the stellar mass, in order to make angular momentum conservation exact. Among other things, this allows users to judge if the precision requirements they have specified is sufficient for the evolution they are calculating.

Our model makes a distinction between low mass stars, which have appreciable surface convective zones, and high mass stars, which do not.

For low mass stars $\left(M_{*}<M_{*}^{\text {crit }}\right)$, the tidal dissipation is assumed to occur in the surface convective zone, and the angular momentum is deposited 
only in that zone, while for high mass stars no such splitting is made.

Furthermore, measurements of stellar spins in open clusters reveal that low mass stars lose most of their initial angular momentum over their lifetime. This is thought to be due to stellar winds which are coupled to the star via its magnetic field. This effect has been extensively studied (c.f. Schatzman 1962; Skumanich 1972; Kawaler 1988; Irwin \& Bouvier 2009; Gallet \& Bouvier 2013, among many others), but remains poorly understood. A combination of theory and observation motivates our formulation of these effects (Stauffer \& Hartmann 1987; Kawaler 1988; Barnes \& Sofia 1996):

$\dot{L}_{\text {wind }} \equiv-K \omega_{\text {surf }} \min \left(\omega_{\text {surf }}, \omega_{\text {sat }}\right)^{2}\left(\frac{R_{*}}{R_{\odot}}\right)^{1 / 2}\left(\frac{M_{*}}{M_{\odot}}\right)^{-1 / 2}$

Finally, Irwin et al. (2007); Irwin \& Bouvier (2009); Denissenkov (2010), argue that in order to explain the observed rotation rates in open clusters of different ages, it is necessary to allow the cores and envelopes of low mass stars to spin at different rates but that they are coupled on a timescale of a few Myr. Our model uses a formulation by MacGregor (1991) and Allain (1998) according to which the rate of angular momentum transfer from the core to the envelope is given by:

$$
\dot{L}_{\text {coup }} \equiv \frac{\Delta L}{\tau_{c}}-\frac{2}{3} R_{\text {rad }}^{2} \omega_{\text {conv }} \dot{M}_{\text {rad }}
$$

where

$$
\Delta L \equiv \frac{I_{\mathrm{conv}} L_{\mathrm{rad}}-I_{\mathrm{rad}} L_{\mathrm{conv}}}{I_{\mathrm{conv}}+I_{\mathrm{rad}}}
$$

For high mass stars $\left(M_{*}>M_{*}^{\text {crit }}\right)$, observations suggest that the angular momentum loss is not important (c.f. Kraft 1967; Zorec \& Royer 2012). Nonetheless, in the interest of generality, we still use Eq. 3 to describe angular momentum loss for high mass stars, albeit with different value of $K$, which can be set to zero if no angular momentum loss should occur.

In our model, high mass stars are not split into zones, but rather solid body rotation is assumed for the star. As a result the rotational evolution simplifies to:

$$
\dot{L}_{*}=\dot{L}_{\text {wind }}+\dot{L}_{\text {tide }}
$$

Finally, we follow the evolution of the stellar quantities $\left(R_{*}, I_{\text {conv }}, I_{\text {rad }}, R_{\text {rad }}\right.$ and $\left.M_{\text {rad }}\right)$ as the stellar structure evolves. Further, $Q_{*}$ can be an arbitrary function of the tidal frequency (the difference between the orbital and stellar spin frequencies). Details on how users can set the frequency dependence of $Q_{*}$ are given in Appendix $\mathrm{A}$. As described in the next section, these basic equations are re-formulated and combined in some cases to facilitate and stabilize the numerical scheme.

\section{Numerical Scheme}

\subsection{Stopping Conditions}

Because Equations 1 and 3 have discontinuities $\left(\right.$ when $\omega_{\text {surf }}=\omega_{\text {orb }}$ for Eq. 1 and when $\omega_{\text {surf }}=\omega_{\text {sat }}$ for Eq. 3), it is beneficial to detect when the evolution goes through these discontinuities and ensure that it does not simply jump over such points, but lands exactly on them (to some precision of course). Such special treatment allows the evolution to be calculated both more accurately and more efficiently.

To see this, consider the discontinuity in Eq. (1. Because the sign of the tidal evolution changes when the spin of the star goes through synchroneity with the orbit, it is possible to lock the system in a state where $\omega_{\text {surf }}$ is held equal to $\omega_{\text {orb }}$. If we simply let the ordinary differential equation (ODE) solver handle this for us, the best possible outcome would be to take tiny steps, oscillating between super- and sub-synchronous rotation. However, if we go through the effort of detecting this and stopping the evolution precisely at the point where synchronous rotation is achieved, we can switch to a different system of differential equations that assumes a spin-orbit lock and uses it to eliminate one of the evolution variables. This avoids the oscillatory behavior, and large time steps can safely be taken.

The spin-orbit lock may not persist indefinitely. The orbit continues to evolve since the system is losing angular momentum due to the stellar wind. Consequently, there may come a point when the tidal dissipation in the star cannot drain sufficient amount of angular momentum from the orbit to compensate for the wind loss and the extra spin up required of the star in order to match the shorter orbital period, at which point the evolution has to revert back to the non-locked equations. 
Next, consider the discontinuity in Eq. 3. Because in this case the rate of evolution of the angular momentum of the convective region (or total angular momentum for the case of a high mass star) is not discontinuous, but its derivative is, the ODE solver can blindly jump over the $\omega_{\text {surf }}=\omega_{\text {sat }}$ point resulting in the change from saturated to non-saturated wind (or vice-versa) happening later than it should. If on the other hand, we detect this and force the solver to land precisely on the critical point, the calculated evolution will be more precise.

In addition to the above discontinuities, we have several others, which are due to the fact that we may want to include parts of the evolution of the system before and after the planet is present.

In the present version of the code, we are able to start the evolution when the protoplanetary disk is still present, assuming that the stellar surface rotation is locked to the rotation rate of the inner edge of the disk (Lin et al. 1996). Then at some specified age, the disk is removed (releasing the surface rotation rate of the star from the lock) and replaced with a planet in a circular orbit. We follow the evolution until either the star leaves the main-sequence or the orbit shrinks so much that the planet is tidally disrupted or engulfed by the star, a condition we refer to as "planet death".

The semimajor axis at which the planet is assumed to be tidally disrupted is calculated according to Roche (1850, 1851):

$$
a_{\text {roche }}=2.44 r_{p}\left(\frac{M_{*}}{m_{p}}\right)^{\frac{1}{3}}
$$

and the planet is assumed to be engulfed by the star when the semimajor axis is equal to the stellar radius.

If the planet dies before the star leaves the main-sequence, the angular momentum of the planetary orbit at the moment of death is added to the stellar convective zone for low mass stars or to the angular momentum of the entire star for high mass stars. We then follow the subsequent rotational evolution of the star until the end of its main sequence phase.

In addition, we allow for user-defined stopping conditions (some function of the orbital parameters and age which are either interesting to the user or indicate that the evolution equations must be modified). Each Stopping Condition should be a quantity that varies smoothly with the evolution (continuous and continuously differentiable up to at least third order) and is zero exactly when the evolution should be stopped. See Appendix B for a detailed description of how stopping conditions are handled. Pre-defined stopping conditions handle all the discontinuities in the evolution equations, and users can define additional stopping conditions as functions of the orbital and system parameters, without needing to understand any of the implementation details. Appendix $\mathrm{A}$ gives details on how that can be achieved.

\subsection{Evolution Modes}

As discussed above, discontinuities in the evolution require switching between different systems of differential equations when some StoppingCondition is encountered. The system of differential equations to use at any given time is determined by an evolution mode and the wind saturation state.

\subsubsection{Wind Saturation States}

The wind saturation state only affects how $\dot{L}_{\text {wind }}$ is calculated.

NOT_SATURATED

$$
\dot{L}_{\text {wind }}=-K \omega_{\text {surf }}^{3}\left(\frac{R_{*}}{R_{\odot}}\right)^{1 / 2}\left(\frac{M_{*}}{M_{\odot}}\right)^{-1 / 2}
$$

\section{UNKNOWN}

$\dot{L}_{\text {wind }}=-K \omega_{\text {surf }} \min \left(\omega_{\text {surf }}, \omega_{\text {sat }}\right)^{2}\left(\frac{R_{*}}{R_{\odot}}\right)^{1 / 2}\left(\frac{M_{*}}{M_{\odot}}\right)^{-1 / 2}$

$$
\begin{aligned}
& \text { SATURATED } \\
& \dot{L}_{\text {wind }}=-K \omega_{\text {surf }} \omega_{\text {sat }}^{2}\left(\frac{R_{*}}{R_{\odot}}\right)^{1 / 2}\left(\frac{M_{*}}{M_{\odot}}\right)^{-1 / 2}
\end{aligned}
$$

The UNKNOWN state never actually occurs during evolution, but is useful when defining stopping conditions for example. 


\subsubsection{Stellar Rotation and Orbital Evolution Modes}

Depending on the stellar rotation evolution mode, the rotational and orbital evolution are calculated using different sets of variables and equations governing their evolution.

\section{LOCKED_TO_DISK}

This is the evolution mode for a system for which the protoplanetary disk is still present. In this case, the spin of the surface convective zone is held at some prescribed constant value $\omega_{\text {disk }}$, representing the orbital frequency of the inner edge of the protoplanetary disk, to which the stellar surface rotation is locked.

In this case, the only evolution variable is $L_{\mathrm{rad}}$, and the equation governing its evolution is:

$$
\dot{L}_{\mathrm{rad}}=-\dot{L}_{\text {coup }}
$$

Where $L_{\text {conv }}$ in Eq. 4 is replaced by $I_{\text {conv }} \omega_{\text {disk }}$.

The reason for including this extra stage is that it makes it possible to start the evolution at the age when the radiative core first begins to form, thus not requiring an initial value to be supplied for its angular momentum (or spin frequency). Instead it acquires angular momentum through core-envelope coupling.

The evolution will switch out of this mode at a prescribed disk-dissipation age. The subsequent evolution mode is FAST_PLANET, LOCKED_TO_PI or SLOW_PLANET depending on the initial semimajor axis at which the planet appears.

This evolution mode only makes sense for low mass stars, since for high mass stars there are no variables left to evolve.

\section{FAST_PLANET}

This is the evolution mode for a system in which the orbital period is shorter than the spin period of the stellar surface. In this case the evolution variables are: $a^{6.5}, L_{\mathrm{conv}}$ and $L_{\mathrm{rad}}$ for low mass stars and $a^{6.5}$ and $L_{*}$ for high mass stars. The equations for their evolution are:

$$
\begin{aligned}
\frac{d a^{6.5}}{d t} & =-\frac{117}{4} \sqrt{\frac{G}{M_{*}}} R_{*}^{5} \frac{m_{p}}{Q_{*}} \\
\dot{L}_{\text {conv }} & =\dot{L}_{\text {coup }}+\dot{L}_{\text {wind }}+\dot{L}_{\text {tide }} \\
\dot{L}_{\text {rad }} & =-\dot{L}_{\text {coup }} \\
\dot{L}_{*} & =\dot{L}_{\text {wind }}+\dot{L}_{\text {tide }}
\end{aligned}
$$

where Equations 13 and 14 are used for low mass stars and eq. 15] for high mass stars.

The reason for using $a^{6.5}$ instead of $a$ as the evolution variable is evident from the first equation above. The rate at which $a^{6.5}$ evolves is independent of $a$. In fact, for a constant $Q_{*}$ it only changes due to $R_{*}$ evolving. This allows the ODE solver to take much larger steps when the orbit has shrunk than would otherwise be possible.

This evolution mode can end in one of two ways:

1. The planet dies, and the subsequent evolution mode is NO_PLANET.

2. The spin period of the stellar surface matches the orbital period, in which case the subsequent evolution mode is either LOCKED_TO_PLANET or SLOW_PLANET, depending on whether the transfer of angular momentum due to tides is sufficient to keep the lock.

\section{LOCKED_TO_PLANET}

This is the evolution mode for a system in which the surface rotation of the star is held locked to the orbit by the dissipation of the stellar tides.

For low mass stars, the evolution variables are $a$ and $L_{\mathrm{rad}}$ and the equations:

$$
\begin{aligned}
\dot{a} & =2 \frac{T-\dot{I}_{\text {conv }} / a}{M_{*} m_{p} /\left(M_{*}+m_{p}\right)-3 I_{\text {conv }} / a^{2}}(16) \\
T & \equiv \sqrt{\frac{a}{\left(M_{*}+m_{p}\right) G}}\left[\dot{L}_{\text {coup }}+\dot{L}_{\text {wind }}\right](17) \\
\dot{L}_{\text {rad }} & =-\dot{L}_{\text {coup }}
\end{aligned}
$$

For high mass stars, the only variable is $a$ and it is evolved according to:

$$
\begin{aligned}
\dot{a} & =2 \frac{T-\dot{I}_{*} / a}{M_{*} m_{p} /\left(M_{*}+m_{p}\right)-3 I_{*} / a^{2}} \\
T & \equiv \sqrt{\frac{a}{\left(M_{*}+m_{p}\right) G}} \dot{L}_{\text {wind }}
\end{aligned}
$$


This evolution mode can end either by the planet dying or by the rate of transfer of angular momentum from the orbit to the star falling below what is required to keep the lock. In the first case, the subsequent evolution mode is NO_PLANET and in the other case it is either FAST_PLANET or SLOW_PLANET.

\section{SLOW_PLANET}

This is the evolution mode for a system in which the orbital period is longer than the spin period of the stellar surface convective zone. In this case the evolution variables are the same as for the FAST_PLANET case: $a^{6.5}, L_{\text {conv }}, L_{\text {rad }}$ for low mass stars and $a^{6.5}, L_{*}$ for high mass stars, and the equations for their evolution are identical, except for a sign change in the equation for the evolution of the semimajor axis.

This evolution mode can end only if The spin period of the stellar surface convective zone matches the orbital period. In which case the subsequent evolution mode is either LOCKED_TO_PLANET or FAST_PLANET, depending on whether the transfer of angular momentum due to tides is sufficient to keep the lock.

\section{NO_PLANET}

This is the evolution mode for a star without a planet in orbit and no protoplanetary disk. Usually this state is reached after the planet dies. The evolution variables are $L_{\text {conv }}$ and $L_{\text {rad }}$ for low mass stars and $L_{*}$ for high mass stars. Their evolution is given by:

$$
\begin{aligned}
\dot{L}_{\text {conv }} & =\dot{L}_{\text {coup }}+\dot{L}_{\text {wind }} \\
\dot{L}_{\text {rad }} & =-\dot{L}_{\text {coup }} \\
\dot{L}_{*} & =\dot{L}_{\text {wind }}
\end{aligned}
$$

This evolution mode persists until the end of the star's lifetime.

\section{Stellar Evolution}

Because the goal of P.O.E.T. is to calculate evolution as efficiently as possible, stellar evolution is handled by interpolating among a pre-computed grid of tracks based on the YREC stellar evolution model (Demarque et al. 2008). In addition, there is a mechanism for users to supply a custom stellar evolution track.
The built-in YREC tracks are for solar metallicity stars with masses $0.5,0.6,0.7,0.8,0.9,1$, $1.05,1.1,1.15$ and 1.2 solar masses. There are two main limitations to this grid: no track extends beyond an age of 10 Gyr or past the point that the star turns off the main sequence. Also, tracks are available only for solar metallicity stars. For this grid, the value for a particular stellar quantity at a given age and stellar mass is calculated by first interpolating each of the grid tracks to a scaled value of the desired age and then interpolating among the values to the desired stellar mass. For a complete description of the interpolation algorithm, see Appendix C.

We employ cubic splines from the $A L G L I B$ library 2 to smooth the evolutionary tracks and tame numerical artifacts that would otherwise result from our use of second derivatives. We then interpolate between tabulated time steps in the stellar evolution tracks to the desired times. While this approach results in robust and fast interpolation, it takes quite a long time to actually derive the smoothing splines. In order to avoid this overhead every time P.O.E.T. is run, the interpolation can be derived once for a grid and "serialized" (saved) to a file using the boost_serialization library (see Appendix A) for future re-use.

It is possible for users to provide an individual track to use as the stellar evolution model if their application requires it. The track is assumed to be applicable for all stars in a run, and only interpolation in age is used. Since, similarly to the tracks included with P.O.E.T., numerical artifacts can result in unusable interpolations, care must be taken when deriving interpolations for user supplied tracks. The best policy is to output and examine the interpolated quantities as well as their first and second derivatives by eye, and adjust the smoothing parameters for the interpolation if necessary.

User supplied tracks can be "serialized" for future re-use just like the built-in grids.

\section{Example Calculations}

Including the evolution of the stellar interior and rotation in a self-consistent way results in much richer behavior, depending on the param-

2 http://www.alglib.net/interpolation/leastsquares.php\#splinefit 


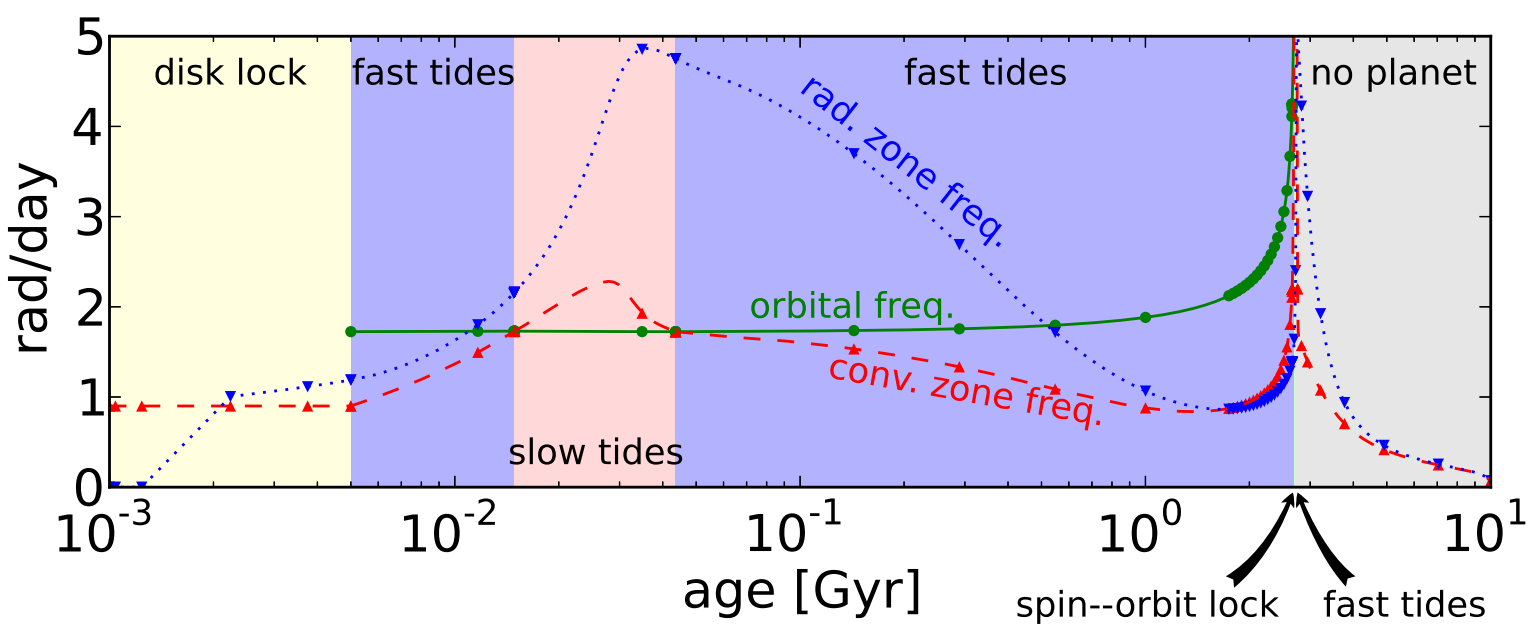

Fig. 1. - The orbital evolution of a 10 Jupiter mass planet around a solar mass star. The lines show the evolution with an imposed maximum time step of $0.1 \mathrm{Myr}$ and the symbols show the time steps taken by our code when no limit is imposed. The line styles and symbols show the evolution of the orbital angular velocity (solid green line and green circles), the angular velocity of the convective envelope of the star (dashed red line and red upward triangles) and the angular velocity of the radiative core of the star (dotted blue line and blue downward triangles). The background denotes the various evolution modes detected by P.O.E.T. From left to right: the convective zone is locked to a disk with a prescribed angular velocity; the planet forms in an orbit faster than the surface spin of the star; the star spins up due to shrinking, exceeding the orbital angular velocity; the stellar wind removes sufficient angular momentum from the star so that its surface rotation drops below the orbital frequency again; the transfer of angular momentum from the orbit to the star synchronizes and locks the surface rotation of the star to the orbit; the lock is broken and the star spins slower than the planet, and finally the planet spirals into the star and deposits its angular momentum resulting in very fast stellar spin which decays due to the stellar wind.

eters of the system being evolved. As an illustration, in Fig. 1] we show the evolution of a 10 Jupiter mass planet around a solar mass star. The values of $Q_{*}$ and the initial semimajor axis were chosen such that the resulting evolution goes through all evolution modes. The stellar wind and core-envelope coupling were left at their default values. Both the lines and points were calculated using P.O.E.T. The only difference is that for the lines the maximum time steps was limited to $0.1 \mathrm{Myr}$, while the symbols show the optimal time steps chosen by the code with no limit imposed, thus demonstrating the large time steps P.O.E.T. is able to take without sacrificing accuracy in the computed evolution. As a reference, computing the evolution with automatic step size control required about one second (on a run-ofthe-mill desktop), most of which was taken up by reading in the "serialized" stellar evolution), while the finely sampled calculation took close to $20 \mathrm{sec}-$ onds, clearly dominated by actually computing the evolution.

Following the evolution of the stellar properties along with the evolution of the orbit allows studies that are otherwise impossible. Penev et al. (2012) modelled detection probabilities for tidally evolving extrasolar planets around evolving stars during the whole stellar main sequence. This calculation incorporated detection biases and provided limits on the tidal dissipation parameter $Q_{*}$ for the host stars from the observed distribution of exoplanet orbital periods.

Zhang \& Penev (2013) suggested using the fact that stars spin up to extremely short periods if they accrete a hot Jupiter to select candidate stars for which this may have occurred. The ability to follow the evolution of the stellar spin was clearly crucial in estimating the prevalence of such fast rotators.

Including the stellar evolution is particularly important for systems like HAT-P-2 (Bakos et al. 2007), HAT-P-20 and HAT-P-21 (Bakos et al. 2011), and WASP-10 (Christian et al. 2009), for which the planets' orbital angular momenta are comparable to the host stars' spin angular momentum. For these cases, tidal interactions can 
temporarily synchronize the stellar spins before the planets finally plunge into their host stars. In Fig. 2 we show the evolution of the orbit of HATP-20b for $Q_{*}=10^{5}$ calculated using three sets of assumptions:

- the full evolution of the stellar structure and rotation, including angular momentum loss to the stellar wind, asynchronous rotation between the stellar radiative core and convective envelope, and starting the star with reasonable initial rotation (solid blue curve).

- assuming solid body rotation for the star, ignoring the loss of angular momentum to stellar wind, and starting the star without any rotation at $5 \mathrm{Myr}$ (dashed green curve).

- ignoring both the rotation and the evolution of the star, and simply calculating the evolution of the semimajor axis (dotted red curve).

In each case, the initial conditions were tuned in order to make the orbital period attain its presently observed value at a system age of 2.9 Gyr (see table 1). The age was chosen because at that age our stellar evolution reproduces the nominal radius of HAT-P-20, and it is consistent (within the uncertainties) with the value quoted in Bakos et al. (2011).

The simplest possible assumptions (assuming nothing about the star changes) do very well at reproducing the future HAT-P-20 orbit. This is because, even though the orbit has much more angular momentum than necessary in order to synchronize the star, after synchronization, the star spins so fast, that it loses angular momentum to its wind at a very high rate. As a result, the tidal dissipation we assumed is just barely large enough to hold the spin-orbit lock, and the orbital evolution proceeds at almost the same rate as under the assumption of a non-rotating star. The early evolution is quite different, due to the fact that for the full evolution, for ages between 25 and 440 Myr, the stellar spin period is shorter than the orbital period (due to the star shrinking onto the main sequence). This results in the planet actually being pushed away from the star during this period.

This calculation illustrates an important result: even after tidal interactions synchronize the

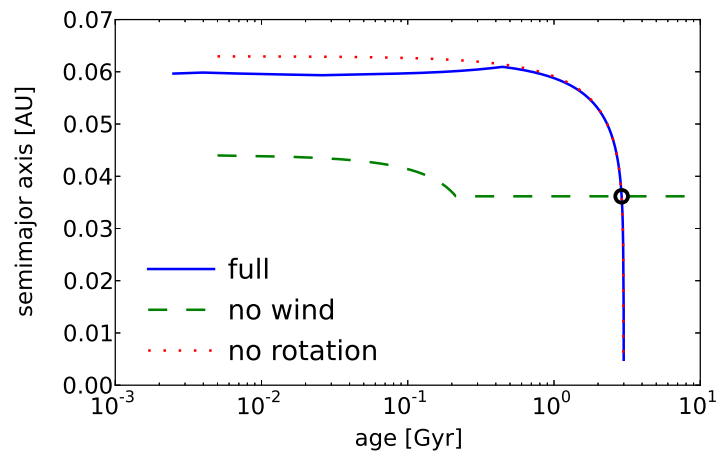

Fig. 2. - The evolution of HAT-P-20b's orbit for $Q_{*}=10^{5}$ including all the details P.O.E.T. is capable of following (solid blue curve), ignoring the evolution of the star, the magnetic wind and core-envelope decoupling (dashed green curve) and fully ignoring any evolution and assuming no rotation for the parent star (dotted red curve). The black circle where all three lines intersect denotes the observed orbit at the assumed present age of the system.

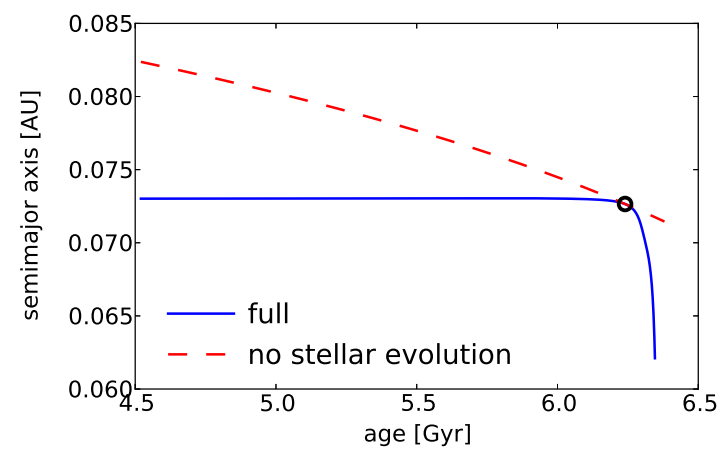

Fig. 3. - The evolution of KOI-2133.01's orbit for $Q_{*}=10^{8}$ including all the details P.O.E.T. is capable of following (solid blue curve), and ignoring the evolution of the star (dashed red curve). The black circle where the two lines touch denotes the observed orbit at the assumed present age of the system. 


\begin{tabular}{r|c|c|c|c|c} 
& \multicolumn{3}{|c}{ HAT-P-20b } & \multicolumn{2}{c}{ KOI-2133 } \\
& full & no wind & no rotation & full & no stellar evolution \\
\hline$K\left[M_{\odot} R_{\odot}^{2}\right.$ day $\left.^{2} \mathrm{rad}^{-2} \mathrm{Gyr}^{-1}\right]$ & 0.17 & 0 & $\infty$ & 0 & 0 \\
$\omega_{\text {sat }}[\mathrm{rad} / \mathrm{day}]$ & 2.45 & 2.45 & 2.45 & $\mathrm{~N} / \mathrm{A}$ & $\mathrm{N} / \mathrm{A}$ \\
$\tau_{c}[\mathrm{Myr}]$ & 28 & 0 & 0 & $\mathrm{~N} / \mathrm{A}$ & $\mathrm{N} / \mathrm{A}$ \\
$Q_{*}$ & $10^{5}$ & $10^{5}$ & $10^{5}$ & $10^{8}$ & $10^{8}$ \\
$M_{*}\left[M_{\odot}\right]$ & 0.756 & 0.756 & 0.756 & 1.31 & 1.31 \\
$m_{p}\left[M_{\text {jup }}\right]$ & 7.3 & 7.3 & 7.3 & 0.88 & 0.88 \\
$r_{p}\left[R_{\text {jup }}\right]$ & 0.876 & 0.876 & 0.876 & 1.384 & 1.384 \\
initial stellar spin period [days] & 7 & $\infty$ & $\infty$ & 6.0 & $\infty$ \\
planet appearance age [Myr] & 5 & 5 & 5 & 4520 & 4520 \\
initial orbital period [days] & 5.96383 & 3.85693 & 6.604712 & 6.29362 & 7.541190
\end{tabular}

Table 1: The values for the various model parameters used to calculate the evolutions of HAT-P-20b's and KOI-2133's orbits

spin of a host star, the planet may still die. Levrard et al. (2009) pointed out that planetary systems with sufficiently large total angular momenta (orbital + spin angular momenta) are formally stable against tidal decay. However, as suggested by Levrard et al. (2009); Barker \& Ogilvie (2009), the continual loss of angular momentum through the stellar wind, included in this study, may doom such planetary systems anyway. In fact, since the rate of angular momentum loss increases with stellar spin, spin synchronization usually results in a tidal decay rate very similar to that computed by assuming the star is not rotating.

Another class of systems which absolutely require the simultaneous calculation of the stellar evolution along with the orbit are planets around evolved stars, like HD 102956b (Johnson et al. 2010), HIP 13044b (Klement et al. 2011) and KOI-2133.01 (Lillo-Box et al. 2013). For these systems, the timescale on which the parent star evolves is comparable to, or even shorter than the timescale for orbital evolution.

Figure 3 demonstrates the difference between including and not the stellar evolution when calculating the orbital evolution of KOI-2133.01. Since the built-in YREC tracks do not go past the end of the main sequence lifetime of any star and do not extend to masses as high as 1.31 (KOI2133's mass), we used MESA (Paxton et al. 2011, 2013) to generate a short post-main-sequence track suitable for this system, which was passed to P.O.E.T. as a custom stellar evolution.
We found that the stellar properties quoted in Lillo-Box et al. (2013) match our track best for an age of 6.24 Gyr (consistent with the system age quoted in the paper to within the uncertainty), so we adopted a present system age of 6.24 Gyr. As before, we tuned the initial conditions (see table 11) to match the presently observed state of the system. As expected, the simplified calculation over-predicts the rate of orbital evolution at early times, because it overestimates the radius of the star, and under-predicts the evolution at later times, since it underestimates the stellar radius.

\section{Conclusions}

We have presented a code (P.O.E.T.) capable of calculating the evolution of extrasolar planet orbits taking into account the rotation and the evolution of the structure of the parent star, including the transfer of angular momentum from the orbit to the star and the loss of angular momentum by the star to a magnetically launched wind.

Because it properly handles all these effects, unlike previous models, P.O.E.T. is capable of following the evolution of planetary orbits from before the star lands on the main sequence to well after it leaves it. Also it properly takes into account the possibility that planets may spin-up their star to synchronous rotation with the orbit, but that this does not mean that further evolution stops.

As any model, P.O.E.T. has limitations. First, at present P.O.E.T. is limited to calculating the evolution of only circular orbits perfectly aligned 
with the stellar equator. This precludes investigations into some of the formation scenarios for hot Jupiters, which predict that exoplanet orbits start with significant eccentricities and/or are significantly inclined with respect to the stellar equator. We plan to address this in two steps: i) implement evolving inclined orbits following the formalism of Lai (2012), ii) introduce eccentricity, by extending that formalism. The first step is a relatively straight forward extension of the current implementation, which simply requires adding more parameters (the seven dissipation efficiencies, or equivalently tidal quality factors, introduced in Lai (2012). The second step is more involved, since for eccentric orbits the planet experiences time variable tidal forces, no matter its spin. This means that a complete calculation must include the dissipation in the planet as well as the star. Further, since unlike for stars, the rate at which energy is deposited in a planet by the tidal dissipation may be important, or even completely dominate the energy budget of the planet, its effects on the planetary structure must be included (cf. Miller et al. 2009).

Second, the grid of stellar evolution models presently included is limited. On the one hand, no tracks extend past the end of the main sequence, and in fact for stars with mass lower than $1 M_{\odot}$ tracks are terminated at 10 Gyr. Further, only tracks for solar metallicity stars are available. Since we provide a mechanism for users to supply their own stellar evolution tracks, these limitations can be easily overcome, as we demonstrated by calculating the evolution of KOI-2133.01's orbit (see Sec. 5). Nevertheless, We are currently in the process of generating more extended grids of stellar evolution models using the MESA suite, which will also be made publicly available.

\section{REFERENCES}

Allain, S. 1998, A\&A, 333, 629

Bakos, G. Á., Hartman, J., Torres, G., Latham, D. W., Kovács, G., Noyes, R. W., Fischer, D. A., Johnson, J. A., Marcy, G. W., Howard, A. W., Kipping, D., Esquerdo, G. A., Shporer, A., Béky, B., Buchhave, L. A., Perumpilly, G., Everett, M., Sasselov, D. D., Stefanik, R. P., Lázár, J., Papp, I., \& Sári, P. 2011, ApJ, 742, 116
Bakos, G. Á., Kovács, G., Torres, G., Fischer, D. A., Latham, D. W., Noyes, R. W., Sasselov, D. D., Mazeh, T., Shporer, A., Butler, R. P., Stefanik, R. P., Fernández, J. M., Sozzetti, A., Pál, A., Johnson, J., Marcy, G. W., Winn, J. N., Sipőcz, B., Lázár, J., Papp, I., \& Sári, P. 2007, ApJ, 670, 826

Barker, A. J. \& Ogilvie, G. I. 2009, MNRAS, 395, 2268

Barnes, S. \& Sofia, S. 1996, ApJ, 462, 746

Batygin, K. \& Adams, F. C. 2013, ApJ, 778, 169

Carlberg, J. K., Majewski, S. R., \& Arras, P. 2009, ApJ, 700, 832

Chambers, J. E. 2009, Annual Review of Earth and Planetary Sciences, 37, 321

Christian, D. J., Gibson, N. P., Simpson, E. K., Street, R. A., Skillen, I., Pollacco, D., Collier Cameron, A., Joshi, Y. C., Keenan, F. P., Stempels, H. C., Haswell, C. A., Horne, K., Anderson, D. R., Bentley, S., Bouchy, F., Clarkson, W. I., Enoch, B., Hebb, L., Hébrard, G., Hellier, C., Irwin, J., Kane, S. R., Lister, T. A., Loeillet, B., Maxted, P., Mayor, M., McDonald, I., Moutou, C., Norton, A. J., Parley, N., Pont, F., Queloz, D., Ryans, R., Smalley, B., Smith, A. M. S., Todd, I., Udry, S., West, R. G., Wheatley, P. J., \& Wilson, D. M. 2009, MNRAS, 392, 1585

Collins, K. A., Eastman, J. D., Beatty, T. G., Siverd, R. J., Gaudi, B. S., Pepper, J., Kielkopf, J. F., Johnson, J. A., Howard, A. W., Fischer, D. A., Manner, M., Bieryla, A., Latham, D. W., Fulton, B. J., Gregorio, J., Buchhave, L. A., Jensen, E. L. N., Stassun, K. G., Penev, K., Crepp, J. R., Hinkley, S., Street, R. A., Cargile, P., Mack, C. E., Oberst, T. E., Avril, R. L., Mellon, S. N., McLeod, K. K., Penny, M. T., Stefanik, R. P., Berlind, P., Calkins, M. L., Mao, Q., Richert, A. J. W., DePoy, D. L., Esquerdo, G. A., Gould, A., Marshall, J. L., Oelkers, R. J., Pogge, R. W., Trueblood, M., \& Trueblood, P. 2013, ArXiv e-prints

Debes, J. H. \& Jackson, B. 2010, ApJ, 723, 1703

Demarque, P., Guenther, D. B., Li, L. H., Mazumdar, A., \& Straka, C. W. 2008, Ap\&SS, 316, 31 
Denissenkov, P. A. 2010, ApJ, 719, 28

Fabrycky, D. \& Tremaine, S. 2007, ApJ, 669, 1298

Gallet, F. \& Bouvier, J. 2013, A\&A, 556, A36

Goldreich, P. 1963, MNRAS, 126, 257

Irwin, J. \& Bouvier, J. 2009, in IAU Symposium, Vol. 258, IAU Symposium, ed. E. E. Mamajek, D. R. Soderblom, \& R. F. G. Wyse, 363-374

Irwin, J., Hodgkin, S., Aigrain, S., Hebb, L., Bouvier, J., Clarke, C., Moraux, E., \& Bramich, D. M. 2007, MNRAS, 377, 741

Jackson, B., Barnes, R., \& Greenberg, R. 2009, ApJ, 698, 1357

Jackson, B., Greenberg, R., \& Barnes, R. 2008, ApJ, 678, 1396

Jackson, B., Miller, N., Barnes, R., Raymond, S. N., Fortney, J. J., \& Greenberg, R. 2010, MNRAS, 407, 910

Johnson, J. A., Bowler, B. P., Howard, A. W., Henry, G. W., Marcy, G. W., Isaacson, H., Brewer, J. M., Fischer, D. A., Morton, T. D., \& Crepp, J. R. 2010, ApJ, 721, L153

Kaula, W. M. 1968, An introduction to planetary physics - The terrestrial planets, ed. Kaula, W. M.

Kawaler, S. D. 1988, ApJ, 333, 236

Klement, R. J., Setiawan, J., Henning, T., Rix, H.-W., Rochau, B., Rodmann, J., \& SchulzeHartung, T. 2011, in IAU Symposium, Vol. 276, IAU Symposium, ed. A. Sozzetti, M. G. Lattanzi, \& A. P. Boss, 121-125

Kraft, R. P. 1967, ApJ, 150, 551

Lai, D. 2012, MNRAS, 423, 486

Levrard, B., Winisdoerffer, C., \& Chabrier, G. 2009, ApJ, 692, L9

Lillo-Box, J., Barrado, D., Moya, A., Montesinos, B., Montalbán, J., Bayo, A., Barbieri, M., Régulo, C., Mancini, L., Bouy, H., \& Henning, T. 2013, ArXiv e-prints

Lin, D. N. C., Bodenheimer, P., \& Richardson, D. C. 1996, Nature, 380, 606
Lithwick, Y. \& Wu, Y. 2013, ArXiv e-prints

MacGregor, K. B. 1991, in NATO ASIC Proc. 340: Angular Momentum Evolution of Young Stars, $315^{-+}$

Miller, N., Fortney, J. J., \& Jackson, B. 2009, ApJ, 702,1413

Nagasawa, M., Ida, S., \& Bessho, T. 2008, ApJ, 678,498

Ogilvie, G. I. \& Lin, D. N. C. 2007, ApJ, 661, 1180

Paxton, B., Bildsten, L., Dotter, A., Herwig, F., Lesaffre, P., \& Timmes, F. 2011, ApJS, 192, 3

Paxton, B., Cantiello, M., Arras, P., Bildsten, L., Brown, E. F., Dotter, A., Mankovich, C., Montgomery, M. H., Stello, D., Timmes, F. X., \& Townsend, R. 2013, ApJS, 208, 4

Penev, K., Jackson, B., Spada, F., \& Thom, N. 2012, ArXiv e-prints

Pepper, J., Siverd, R. J., Beatty, T. G., Gaudi, B. S., Stassun, K. G., Eastman, J., Collins, K., Latham, D. W., Bieryla, A., Buchhave, L. A., Jensen, E. L. N., Manner, M., Penev, K., Crepp, J. R., Cargile, P. A., Dhital, S., Calkins, M. L., Esquerdo, G. A., Berlind, P., Fulton, B. J., Street, R., Ma, B., Ge, J., Wang, J., Mao, Q., Richert, A. J. W., Gould, A., DePoy, D. L., Kielkopf, J. F., Marshall, J. L., Pogge, R. W., Stefanik, R. P., Trueblood, M., \& Trueblood, P. 2013, ApJ, 773, 64

Rasio, F. A. \& Ford, E. B. 1996, Science, 274, 954

Roche, E. 1850, Académie des sciences de Montpellier: Mémoires de la section des sciences, 1, 333

- 1851, Académie des sciences de Montpellier: Mémoires de la section des sciences, 2, 333

Sandquist, E. L., Dokter, J. J., Lin, D. N. C., \& Mardling, R. A. 2002, ApJ, 572, 1012

Sasselov, D. D. 2003, ApJ, 596, 1327

Schatzman, E. 1962, Annales d'Astrophysique, 25, 18

Skumanich, A. 1972, ApJ, 171, 565 
Stauffer, J. R. \& Hartmann, L. W. 1987, ApJ, 318, 337

Teitler, S. \& Königl, A. 2014, ArXiv e-prints

Valsecchi, F. \& Rasio, F. A. 2014, ArXiv e-prints

Weidenschilling, S. J. \& Marzari, F. 1996, Nature, 384,619

Zhang, M. \& Penev, K. M. 2013, in preparation

Zorec, J. \& Royer, F. 2012, A\&A, 537, A120

This 2-column preprint was prepared with the AAS IATEX

macros v5.2. 


\section{A. Installation and Usage}

The latest official release of P.O.E.T. can be downloaded from:

http://www.astro.princeton.edu/ kpenev/tidal_orbital_evolution/poet.tgz.

or

$$
\text { https://www.assembla.com/spaces/tidal-orbital-evolution }
$$

In addition, the python module is installable from PyPI - the Python Package Index ${ }^{3}$ (package name POET). The PyPI website provides full instructions on how to install packages hosted there.

Compilation requires the development version (header files as well as shared library files) of the following libraries: gs 14, boost_serialization, and argtable26. Those are available as packages on most Linux and Mac (through macports) distributions. All other libraries needed by the code are already directly included with the source.

After those dependencies are met, compiling and installing the code is simple. After changing to the src sub-directory, run:

sudo make install BINDIR='<desired location of executables>' DATADIR='<desired location of data files>'

This will compile an executable called poet and copy it to the destination you specified with the BINDIR option, and copy P.O.E.T. related data (e.g. stellar evolution tracks) to the destination specified with DATADIR. Finally, it will compile and install in the standard location for your system a python module named poet.

For a full documentation of the available make targets run make help or see:

http://www.astro.princeton.edu/ kpenev/tidal_orbital_evolution/compilation.html.

Successful compilation produces a single executable named poet and places it in the location specified by the BINDIR argument to make install. In addition, P.O.E.T. related data is copied to the location specified by the DATADIR argument. Finally, a python module named poet is compiled and installed in the standard location for your system. Commonly, you would want to make sure that BINDIR is in your search path.

All the parameters that enter into the evolution equations (see Section 2) are changeable through command line options of poet. Rather than listing here the close to 50 command line options, which may change in the future, we outline the general scheme used and for details refer the reader to:

http://www.astro.princeton.edu/ kpenev/tidal_orbital_evolution/usage.html,

which always documents the latest release. The same information can also be obtained by invoking poet $-\mathrm{h}$.

In order to fully define the problem, many parameters need to have their values specified. In addition, many applications will require calculating a large number of orbits for which most parameters are the same, and only a small subset vary. In order to handle these design constraints in the most convenient for the user

3 https://pypi.python.org/pypi

${ }^{4}$ http://www.gnu.org/software/gsl/

5 http://www.boost.org/

${ }^{6}$ http://argtable.sourceforge.net/ 
fashion, we have introduced command line options for all parameters (all of which have "reasonable" default values) and an input file for batch jobs, which lists only the parameters that change between evolutions.

Since all options have default values, the simplest valid command line for running P.O.E.T. is poet. This will produce an output file called poet.evol containing a pre-defined set of columns containing the evolution for a one Jupiter mass planet around a solar mass star with default values for all parameters needed for the evolution.

As noted in the main text, users can change the frequency dependence of the tidal quality factor, as well as define custom stopping conditions. The former requires the users to change a file named: StellarQ.cpp which contains the definitions of $Q_{*}\left(\omega_{\text {orb }}-\omega_{\text {surf }}\right)$ and its derivative. At present, $Q_{*}$ is not allowed to depend on anything except the angular velocity of the tides as seen by the star. Defining custom stopping conditions requires editing: ExternalStoppingConditions.h and ExternalStoppingConditions.cpp. In either case, the user need not know anything about the implementation details of P.O.E.T., but simply define the relevant functions. Both types of modifications require re-compiling.

\section{B. Handling Stopping Conditions}

In P.O.E.T., stopping conditions are simply functions of the system age and state that have a value of zero when the evolution should be stopped (either because there is some discontinuity requiring a change in the differential equations being solved, or because something that a user is interested in has happened). They are assumed to be continuously differentiable at least up to second order. Below we provide the full details of how P.O.E.T. handles stopping conditions internally.

As the orbital evolution is being calculated, the values of the active stopping conditions are stored, and when either a zero crossing or an extremum for which the absolute value of any condition has a minimum is detected, the evolution is forced to land on the zero-crossing or the extremum to within some specified accuracy.

The algorithm used is as follows:

1. For a zero-crossing, if the absolute value of the stopping condition is smaller than some tolerance, the evolution is stopped and the appropriate changes are made to the equations and variables before it is continued. For an extremum, if the stopping condition value is within some tolerance of the estimated extremal value, and still no zero-crossing has been detected, the evolution simply continues.

2. If the last step did not take us close enough to the zero-crossing or the extremum, the evolution is reset to the last point before the event, the time of the extremum or zero-crossing is estimated and another step is taken to the estimated time.

3. If the new point is before the zero-crossing or extremum, it is added to the stored evolution and the evolution is allowed to proceed (knowing it will very shortly be stopped again).

4. If the new point is after the zero-crossing or extremum, we go back to step 1.

Stopping conditions come in two flavors: those for which the first order derivative is available and those for which it is not. In either case, a zero crossing is detected by the change in sign of the stopping condition.

For stopping conditions with derivative information, extrema are detected by a sign change in the first order derivative in two consecutive evolution steps. Extrema are only investigated if the sign of the earlier derivative is opposite the sign of the earlier stopping condition value. In this case, the locations of zerocrossings and extrema, as well as values at the extremum (needed for steps 1 and 2 above) are estimated from the unique third order polynomial that passes through the two points surrounding the event and has the calculated derivatives at those points.

For stopping conditions without derivative information, extrema are detected and investigated by finding a time step for which the stopping condition value is smaller in absolute value than for either of its neigh- 

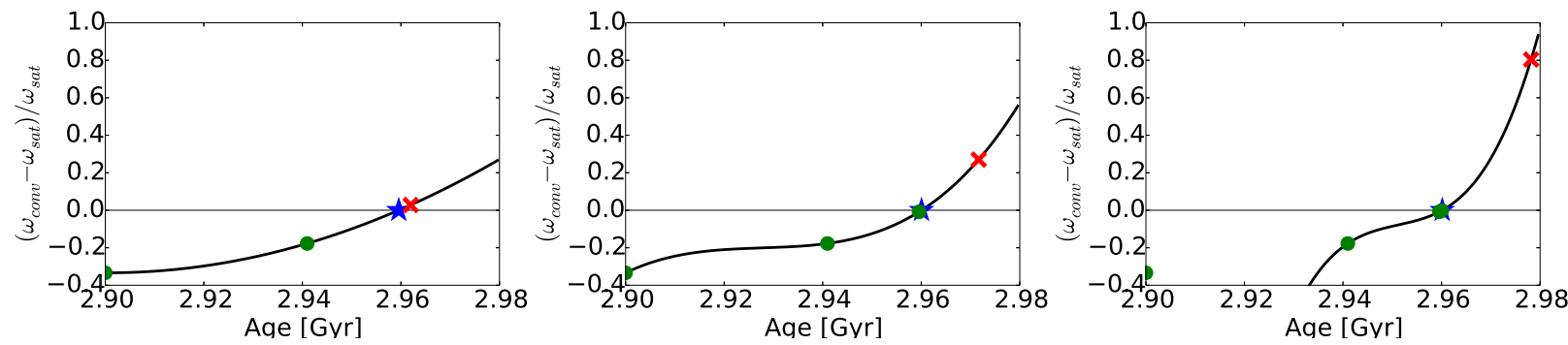

Fig. 4.- The wind saturation stopping condition at work (see text for details). Filled green circles indicate steps that are included in the evolution, red crosses indicate steps that are discarded, the blue star indicates the best estimate for the exact moment when the stopping condition is zero and the black curve shows the polynomial derived from some of the accepted and discarded steps to estimate the zero of the stopping condition.

bors. Without derivative information, zero-crossing, extremum age and stopping condition value are again estimated from a third order polynomial. The coefficients of that polynomial in this case are calculated from four points in the evolution that satisfy the following conditions:

- they are either points from before the event, or are steps of various size started from the last point before.

- the zero-crossing or extremum being investigated occurred somewhere between the first and last point in the sequence.

- the time difference between the first and last points is the shortest possible given the above two constraints.

Figure 4 demonstrates the workings of the wind saturation stopping condition for the HAT-P-20 evolution shown in fig. 2 to detect when the loss of angular momentum to the stellar wind must be switched from its unsaturated to its saturated form (see sec. 3.2.1) as the star is spun-up in the final stages of HAT-P-20b's inspiral. The stopping condition is defined as $\left(\omega_{\text {conv }}-\omega_{\text {sat }}\right) / \omega_{\text {sat }}$, where $\omega_{\text {conv }}$ is the angular frequency at which the stellar convective zone spins, and $\omega_{\text {sat }}$ is the frequency at which the magnetic wind saturates.

The panels in fig. 4 Going from left to right show consecutive steps taken by P.O.E.T. when calculating the evolution. In the first panel, three steps after the last evolution mode change, the wind saturation condition changes sign, from negative (the two green circles) to positive (the red cross). This triggers the stopping condition mechanism. Because only three points are available at this time, a second order polynomial is derived that passes through the three points and is used to estimate the time when the stopping condition is exactly zero (blue star).

In the middle panel, P.O.E.T. has taken a step of the appropriate size to land on the estimated zero and found that the stopping condition is still negative there (the right most green circle at an age of 2.96 Gyr). Because at this point, the stopping condition has not changed sign yet, it is accepted and the previous point which stepped over the sign change is discarded. Then, another step is taken, resulting in a positive value of the wind saturation condition (the red cross at an age of $2.97 \mathrm{Gyr}$ ). Now, four values for the stopping condition are available, so a full third order polynomial is derived passing through all of them, and a new estimate for the location of the zero is derived (the blue star).

In the right panel, a step has been taken to the latest estimate for the location of the zero, resulting in a negative stopping condition value, so it is accepted and another step is taken yielding a positive value. Now P.O.E.T. has 5 points at its disposal to estimate the zero-crossing from (there are actually two green circles very close to each other at an age of $2.96 \mathrm{Gyr}$ ), but since only four can be used to derive the next estimate for the zero crossing (no more than third order polynomials are used), the earliest point (at an age of 2.90 Gyr) is not used. The new polynomial produces yet another estimate for the zero-crossing, and this time 
when P.O.E.T. steps there, the value of the stopping condition is zero to within the specified tolerance, so the point is accepted, the evolution is interrupted and continues with the saturated wind expression from then on.

\section{Stellar Evolution Interpolation}

After experimenting with various algorithms for interpolating over a grid of stellar evolution tracks the following prescription seemed to work best for interpolating some quantity $(q)$ to estimate the value it would take for a star of mass $M_{*}$ at and age of $t\left(q\left(M_{*}, t\right)\right)$ :

1. Starting from a set of tracks calculated using the YREC model, for each track of mass $M_{i}$ a smoothing spline interpolation is derived (or is read from a previously serialized file) giving $q\left[M_{i}, \ln (t)\right]$, where $t$ is the stellar age. This happens at the beginning of the execution of a poet job.

2. At each evaluation, for each stellar track corresponding to mass $M_{i}$ the pre-derived smoothing spline is evaluated to calculate $q_{i} \equiv q\left[M_{i}, \ln \left(t\left(M_{*} / M_{i}\right)^{p}\right)\right]$. By default $p=2.5$, but the value can be changed from the command line or from an input file (see appendix $\mathrm{A}$ ).

3. Derive a non-smoothing (passes exactly through the points) cubic spline of $q_{i}$ versus $M_{i}$ and evaluate it at $M_{*}$.

We scale the ages at which the evolutionary sequences in our YREC grids are evaluated by the stellar mass $\left(M_{i}\right)$ of each sequence because the key stages in stellar evolution take place at different ages for stars of different masses. We found that the particular scaling we use $\left(t\left(M_{*} / M_{i}\right)^{2.5}\right)$ aligns these stages for stars of different masses in a way that optimizes interpolation.

An illustration of how the stellar evolution interpolation works when deriving the value of the stellar radius for a $0.95 M_{\odot}$ star at an age of $28.1 \mathrm{Myr}$ is given in Figure 5 The interpolation age was deliberately chosen to highlight the benefit of scaling the ages at which tracks are evaluated. In the left panel of Fig. 5 we see that near that age the radius of the star exhibits a sharp drop. By scaling the ages at which each track is evaluated, we end up with always using the value of the track right before the feature, leading to the very smooth curve shown in the right panel of the figure. If instead we evaluated all tracks at the desired age of 28.1 Myr, there would be a jump in the radius as a function of mass between 0.9 and $1 M_{\odot}$, which could lead to bad interpolation results. 

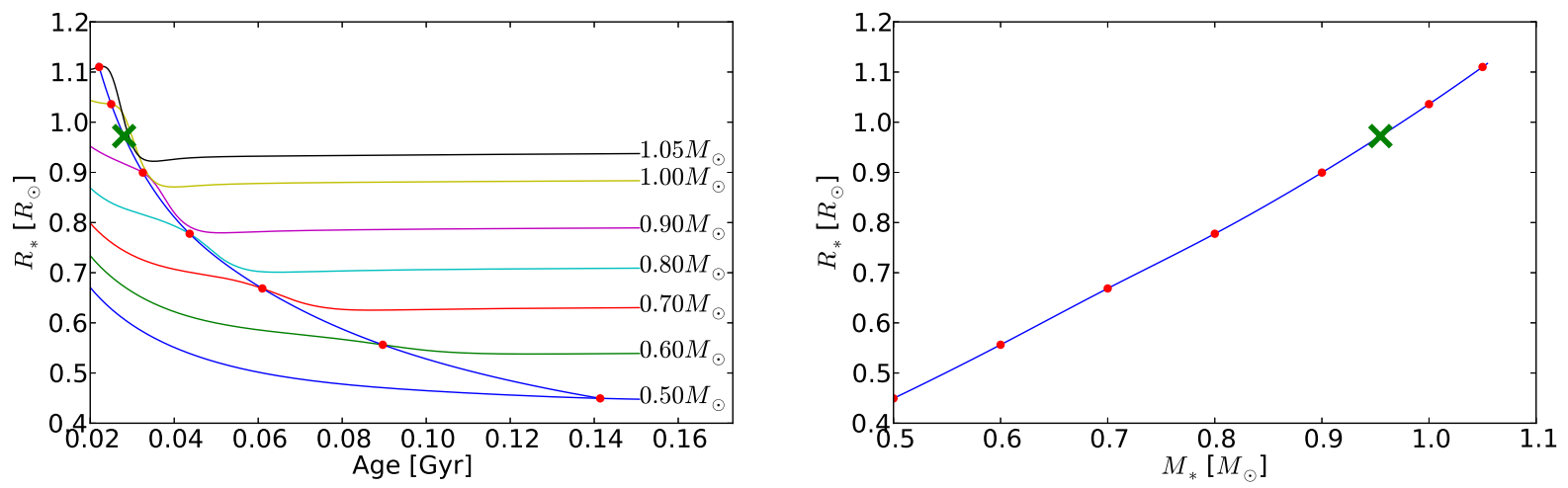

Fig. 5.- An illustration of how interpolating the stellar radius from the YREC tracks works. Left: the lines labeled with stellar masses are the smoothing cubic spline interpolations of the value of the radius $\left(R_{*}\right)$ for each YREC track (step 1); the red circles show the set of points selected in step 2 the curve passing through all the red points is the spline derived by the mass interpolation, where we have assigned an age to each point scaled the same way as the track points were in step 2 Right: the red points are the same red points as in the left plot but plotted against mass instead of age; the curve passing through the points is the cubic spline derived in step 3 In both plots, the green cross shows the final result of the interpolation. 